\title{
Neuromonitor-guided repair of thoracoabdominal aortic aneurysms
}

\author{
Anthony L. Estrera, MD, ${ }^{\mathrm{a}}$ Roy Sheinbaum, MD, ${ }^{\mathrm{a}}$ Charles C. Miller III, PhD, ${ }^{\mathrm{b}}$ Ryan Harrison, BA, ${ }^{\mathrm{a}}$ and \\ Hazim J. Safi, MD ${ }^{\mathrm{a}}$
}

Objective: Monitoring during thoracoabdominal aortic aneurysm repair has included the use of cerebrospinal fluid drainage and motor and somatosensory evoked potentials. We report our experience with neuromonitoring-guided thoracoabdominal aortic aneurysm repair.

Methods: Between November 2008 and January 2010, 105 thoracic aorta repairs were performed; 89\% of patients (93/105) underwent repair using cerebrospinal fluid drainage and distal aortic perfusion. In addition, somatosensory and motor evoked potentials were monitored during repair, and active intraoperative maneuvers were undertaken in response to changes in the signals. Intraoperative maneuvers included intercostal artery reimplantation.

Results: In-hospital mortality for thoracic and thoracoabdominal aortic repair was 5.7\% (6/105). Immediate spinal cord injury occurred in 1 patient $(1 \%)$, and 3 patients $(3 \%)$ had delayed neurologic deficit. Intercostal arteries were reattached in $85 \%$ of possible cases $(51 / 60)$. Somatosensory evoked potentials achieved adequate readings in $99 \%$ of cases (102/103). Loss of somatosensory evoked potential was encountered in $26 \%$ of cases $(27 / 102)$, and return of somatosensory evoked potentials occurred in all cases after intraoperative maneuvers. Motor evoked potentials achieved adequate readings in $96 \%$ of cases (99/103). Loss of motor evoked potential was encountered in $50 \%$ of cases (50/99), and return of motor evoked potentials occurred in all but 1 case $(95 \%)$. This patient awoke with an immediate spinal neurologic deficit.

Conclusions: Neuromonitoring using somatosensory evoked potentials and motor evoked potentials seems useful during thoracoabdominal aortic aneurysm repair. Alterations in intraoperative conduct resulted in return of neuromonitoring signals. This suggests a benefit in intercostal artery reimplantation via increasing perfusion to the collateral network of the spinal cord. Further studies using neuromonitoring-guided repair of thoracoabdominal aortic aneurysms are warranted. (J Thorac Cardiovasc Surg 2010;140:S131-5)

Many advances have been made in the treatment of thoracic and thoracoabdominal aortic aneurysms (TAAA) and dissections. Recently, improved early outcomes have been realized with endovascular therapies for the thoracic aorta, but long-term outcomes remain in question. In addition, the applicability of thoracic endovascular aortic repair (TEVAR) approaches remains limited to those cases with acceptable anatomic criteria, and TEVAR may be contraindicated in cases of genetically associated disorders. For these reasons, open repair remains an essential approach for the aortic specialist.

In specialized centers, results of open repair have improved over the past decade using differing adjuncts for spinal cord protection. Regardless of the adjuncts used, all surgeons recognize the importance of spinal cord protection in preventing the dreaded complication of paraplegia. When

\footnotetext{
From the Cardiothoracic and Vascular Surgery, ${ }^{\text {a }}$ University of Texas Medical School Houston, Memorial Hermann Heart and Vascular Institute, Houston, Tex; and 2-Biomedical Sciences, ${ }^{\mathrm{b}}$ Texas Tech University Medical School, El Paso, Tex. Disclosures: Anthony L. Estrera, Roy Sheinbaum, Charles C. Miller III, Ryan Harrison and Hazim J. Safi have nothing to disclose with regard to commercial support. Received for publication May 12, 2010; accepted for publication July 20, 2010. Address for reprints: Anthony L. Estrera, MD, Suite 2850, 6400 Fannin St, Houston, TX 77030 (E-mail: Anthony.l.estrera@uth.tmc.edu). $0022-5223 / \$ 36.00$

Copyright (c) 2010 by The American Association for Thoracic Surgery doi:10.1016/j.jtcvs.2010.07.058
}

aortic crossclamping is used alone for repair of the most extensive TAAA, the extent II aneurysm, the risk of paraplegia has been reported as high as $50 \%$ when the ischemic time is greater than 45 minutes. ${ }^{1}$

The benefit of neurologic monitoring of spinal cord function, however, remains less established during TAAA repair. ${ }^{2}$ We previously demonstrated that both somatosensory evoked potentials (SSEPs) and motor evoked potentials (MEPs) were relatively insensitive but very specific in detecting spinal cord injury after TAAA repair. ${ }^{3,4}$ A limitation of these studies was that alterations in the conduct of the operation occurred as a result of changes encountered during the procedure. Without a true control group for comparison, the actual sensitivity could not be determined. Nevertheless, their specificity and high negative predictive value still made these monitoring modalities useful.

With increasing experience with SSEPs and MEPs, we have begun to use the intraoperative results of these monitoring modalities to guide the conduct of the operation. This study describes our neuromonitoring-guided approach to TAAA repair and analyzes early results of this approach with a focus on spinal cord injury.

\section{MATERIALS AND METHODS}

The Committee for the Protection of Human Subjects at the University of Texas Houston Medical School approved review of the data collected for this retrospective observational study. 


\section{Abbreviations and Acronyms \\ $\mathrm{CSF}=$ cerebrospinal fluid \\ CSFD = cerebrospinal fluid drainage \\ DAP $=$ distal aortic perfusion \\ ICA $=$ intercostal artery \\ MEP $=$ motor evoked potential \\ SSEP = somatosensory evoked potential \\ TAAA $=$ thoracoabdominal aortic aneurysm \\ TEVAR $=$ thoracic endovascular aortic repair}

Between November 2008 and January 2010, 105 open repairs of the descending thoracic and thoracoabdominal aorta were performed. The mean age was 60 years (range, $17-83$ years), and 38\% (40/105) were women. The distribution of thoracic extent was descending thoracic in $37 \%$, TAAA (extent I) in $10 \%$, TAAA extent II in $15 \%$, extent III in $12 \%$, extent IV in $20 \%$, and extent $\mathrm{V}$ in $6 \%$ of patients. Patient characteristics are summarized in Table 1.

The combined adjuncts, cerebrospinal fluid drainage (CSFD) and distal aortic perfusion (DAP), were used in $89 \%$ of cases (93/105). CSFD was used in $98 \%$ of cases $(103 / 105)$, although in 3 cases, the cerebrospinal fluid (CSF) drain was inserted after the repair was completed. CSFD was not used in cases of rupture, acute trauma, infection, or prior paraplegia.

\section{Neurologic Monitoring}

Our current approach for TAAA repair involves monitoring of CSF pressure and both SSEPs and MEPs. The details of our approach have been described $^{5,6}$ and are briefly reviewed in this article.

\section{Anesthesia \\ Modification of anesthetic technique is required for MEPs and SSEPs. Induction consists of narcotic loading: fentanyl citrate $(15 \mu \mathrm{g} / \mathrm{kg})$, midazo- lam $(0.05 \mathrm{mg} / \mathrm{kg})$, propofol $0.5 \mathrm{mg} / \mathrm{kg}$, and cisatracurium $0.2 \mathrm{mg} / \mathrm{kg}$. Pa- tients are then maintained on a volatile agent (isoflurane) at 0.5 minimal alveolar concentration. Neuromuscular blockade is maintained at $1 / 4$ train-of-4. With this approach, little compromise of neuromotor or neuro- sensory monitoring occurs.}

\section{Somatosensory Evoked Potential and Motor Evoked Potential Monitoring}

A team led by a neurologist/neurophysiologist performed neurophysiologic monitoring. Eight-channel electroencephalogram monitoring was performed during the surgical procedure using a Nicolet Viking Four (Nicolet VikingQuest, Madison, Wis). Evoked potentials were performed with the additional use of a Digitimer generator stimulator (Digitimer, Hertfordshire, UK) for the MEPs. For SSEP monitoring, stimulatory electrodes were placed bilaterally at the level of the malleolus. Recording electrodes were bilaterally placed at 3 levels: popliteal fossa, cervical spine (C5), and vertex. The right and left posterior tibial nerves were stimulated at the ankle to obtain an averaged waveform, repeated every 3 minutes continuously throughout the operation. SSEPs were bilaterally recorded at the 3 levels. A baseline SSEP tracing was obtained before the start of the operation. All subsequent tracings were superimposed and compared with this baseline. The traditional 10/50 rule was considered to define SSEP abnormalities: a $10 \%$ change in latency or a $50 \%$ change in amplitude. The evaluation of 3 channels allowed us to distinguish SSEP changes related to spinal cord injury from peripheral nerve ischemia or cerebral injury.

For MEP monitoring, electrodes were placed at $\mathrm{C} 3$ and $\mathrm{C} 4$ as defined by the International 10-20 system, stimulating the precentral gyrus. The stim- ulus consisted of a train-of-5, an interstimulus interval of $2 \mathrm{msec}$, and voltage as high as 400 volts. Myogenic responses were recorded bilaterally with needle electrodes placed in the abductor digiti minimi, tibialis anterior, and abductor hallucis muscles. Compound muscle action potentials were checked intermittently throughout the operation and considered on an allor-none basis as present or absent.

\section{Operative Approach}

The conduct of the repair depended on the extent of the TAAA. Repair of TAAA extent II encompassed all aspects of our technique and will therefore be described. This approach relies on DAP, CSFD, and moderate hypothermia. DAP is instituted by cannulating the left inferior pulmonary vein for venous drainage and the left common femoral artery, using an 8-mm knitted Dacron graft in a side-to-side fashion. We previously demonstrated the benefit of the side-to-side anastomosis and temperature reduction on warm lower-extremity ischemia and its potential impact on postoperative renal dysfunction. ${ }^{7}$

In addition, sequential clamping, reattachment of patent intercostal arteries (ICAs) T8 to T12, and visceral and renal artery perfusion are used in most cases. Reattachment of ICAs is performed using the island patch approach unless connective tissue disorders are suspected, in which case they are bypassed using a side-to-side approach (loop graft). Premanufactured side-armed branched thoracoabdominal grafts are used for reattachment of the visceral and renal arteries if Marfan or other connective tissue disorders are encountered. Mean aortic clamp time was $52 \pm 36$ minutes, and the mean DAP time was $51 \pm 42$ minutes.

\section{Neuromonitoring-Guided Thoracoabdominal Aortic Aneurysm Repair}

The goal of the operation was to reestablish aortic continuity expeditiously. The conduct of the procedure included the proximal anastomosis with sequential clamping, intercostal reimplantation, visceral and renal reimplantation, and distal anastomosis. During the intercostal phase, if important ICAs were patent but no changes in SSEPs or MEPs occurred, then temporary occlusion with number 3 occlusion balloon catheters in patent ICAs was used, and completion of the visceral and renal reimplantation, and the distal anastomosis were performed. The ICAs were reimplanted only if neurologic signals changed. Debate on the benefit of ICA reattachment remains, ${ }^{8-11}$ but we previously demonstrated the benefit of reattachment of ICAs T8 to T12. Thus, patent important ICAs (ie, T8-T12) were reimplanted if changes on neuromonitoring (SSEPs and MEPs) were encountered. The corrective maneuvers are listed in Table 2. When a signal loss was experienced, the action most closely linked temporally with return of the neuromonitoring signal was considered responsible for the return.

\section{Postoperative Drainage}

SSEPs and MEPs were not monitored postoperatively; thus, only changes in neurologic status dictated CSF drain management. The CSF drain was maintained for 3 days postoperatively on the basis of our previous studies of delayed neurologic deficit. ${ }^{6}$ If the CSF pressure was greater than $10 \mathrm{~mm} \mathrm{Hg}$, CSF was drained to a limit of $15 \mathrm{~mL} / \mathrm{h}$ when the patient was neurologically intact. If delayed neurologic deficit occurred, then CSF was drained to maintain a pressure of less than $5 \mathrm{~mm} \mathrm{Hg}$ without limit under our COPS protocol (Figure 1). This assumed that the CSF remained clear and was not bloody. Because drain malfunction has been identified as a risk factor for delayed neurologic deficit, drain malfunction was actively corrected and the drain was replaced if required. ${ }^{6}$

\section{Statistical Methods and Definitions}

Data were collected prospectively on standardized forms by a trained masters-level nurse researcher. The information was entered into a dedicated database housed on a secure server. Evoked potential measures were coded as "permanent," "transient," or "any" (permanent or transient) change. Permanent change was defined as an absence of signal at the conclusion of surgery. 
TABLE 1. Patient characteristics

\begin{tabular}{lcc}
\hline \multicolumn{1}{c}{ Variable } & $\mathbf{N}$ & $\%(\mathbf{n} / \mathbf{1 0 5})$ \\
\hline Age, $\mathrm{y}$ & $60(17-83)$ & \\
Women & 40 & $38 \%$ \\
Extent & & \\
DTAA & 39 & $37 \%$ \\
TAAA I & 10 & $10 \%$ \\
TAAA II & 16 & $15 \%$ \\
TAAA III & 13 & $12 \%$ \\
TAAA IV & 21 & $20 \%$ \\
TAAA V & 6 & $6 \%$ \\
Dissection & 46 & $44 \%$ \\
Acute & 4 & $4 \%$ \\
Chronic & 43 & $41 \%$ \\
Rupture & 10 & $9 \%$ \\
Marfan & 5 & $5 \%$ \\
Preoperative renal dysfunction & 61 & $58 \%$ \\
Atheromatous disease & 49 & $47 \%$ \\
Preoperative CVA & 5 & $5 \%$ \\
\hline$D T A A$, Descending thoracic aortic aneurysm; $T A A A$, thoracoabdominal aortic aneu- \\
rysm; $C V A$, cerebrovascular accident.
\end{tabular}

\section{RESULTS}

In-hospital mortality for thoracic and thoracoabdominal aortic repair was $5.7 \%(6 / 105)$. Stroke occurred in 1 patient $(1 \%)$. Immediate spinal cord injury occurred in 1 patient $(1 \%)$, and 3 patients $(3 \%)$ had delayed neurologic deficit. Of the 3 patients with delayed spinal cord injury, 2 recovered during the hospitalization. In 2 patients with delayed neurologic deficits, 1 aneurysm repaired was an extent II TAAA and 1 aneurysm was an extent IV TAAA. The one immediate neurologic deficit occurred in a patient with an extent IV TAAA.

\section{Intercostal Artery Status}

By using the approach of reimplanting all patent significant ICAs, 60 patients $(57 \%)$ had aortic repairs in which ICAs T8 to T12 could have been reimplanted, that is, repairs in which the extent of aneurysm was appropriate and the ICAs were patent. Of the 45 patients in whom ICA reimplantation was not applicable, 5 had aneurysms that involved sig-

TABLE 2. Intraoperative changes in response to loss of signals

\begin{tabular}{|c|c|}
\hline \multirow[t]{4}{*}{ Anesthetic maneuvers: } & $\begin{array}{l}\text { Increasing the mean central pressure } \\
\text { to }>80 \mathrm{~mm} \mathrm{Hg}\end{array}$ \\
\hline & $\begin{array}{l}\text { Increasing distal aortic pressure } \\
\text { to }>60 \mathrm{~mm} \mathrm{Hg}\end{array}$ \\
\hline & $\begin{array}{l}\text { Decreasing cerebrospinal fluid pressure by } \\
\text { free gravity drainage }\end{array}$ \\
\hline & Increasing hemoglobin levels by transfusion \\
\hline \multirow[t]{5}{*}{ Surgical maneuvers: } & Immediate reimplantation of $\mathrm{T} 8$ to $\mathrm{T} 12$ \\
\hline & Establish DAP \\
\hline & Establish pulsatile flow to pertinent ICA \\
\hline & Reimplant other patent ICA, T4-T7, L1, L2 \\
\hline & $\begin{array}{l}\text { Complete repair to establish pulsatile flow to } \\
\text { entire "collateral network" }\end{array}$ \\
\hline
\end{tabular}

$D A P$, Distal aortic perfusion; $I C A$, internal carotid artery.

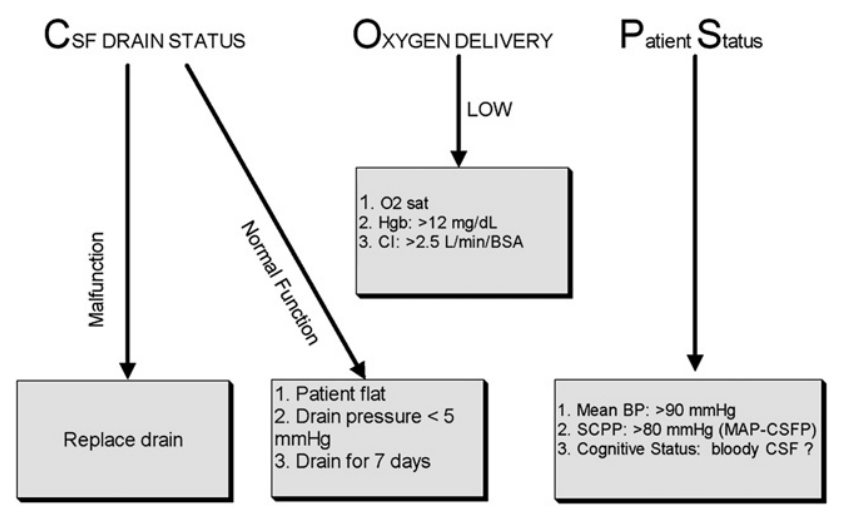

FIGURE 1. Treatment for delayed neurologic deficit: The COPS protocol for managing CSFD. $C S F$, Cerebrospinal fluid; $\mathrm{Hg} b$, hemoglobin; $\mathrm{CI}$, confidence interval; $B S A$, body surface area; $B P$, blood pressure; $S C P P$, increase in spinal cord perfusion pressure; $M A P$, mean arterial pressure; $C S F P$, cerebrospinal fluid pressure.

nificant ICAs that were all occluded, 21 had TAAA extent IV aneurysms without the need for ICA reimplantation, and 19 had aneurysms involving only the proximal descending thoracic artery (extent A), not involving significant ICAs. Of the cohort who could have had ICAs reimplanted, $85 \%(51 / 60)$ underwent reimplantation of ICAs. In 9 patients $(15 \%)$, no changes in neuromonitoring occurred, and subsequent ligation of the significant ICAs was performed.

\section{Somatosensory Evoked Potentials and Motor Evoked Potentials}

SSEPs were elicited in $98 \%$ of cases (103/105), achieving adequate readings in $99 \%$ of attempts (102/103). Loss of SSEPs was encountered in $26 \%$ of cases $(27 / 102)$. Of the patients with SSEP loss, all had return of signals at the conclusion of the operation. Specifically, 48\% (13/27) of those with SSEP loss had return of the signal with ICA reimplantation, 37\% (10/27) with distal pulsatile flow, 7\% (2/27) with DAP, and 7\% (2/27) returned spontaneously without any specific operative measures noted.

MEPs were also performed in $98 \%$ of all cases (103/105), with adequate readings obtained in $96 \%$ of those cases ( $99 /$ 103). Loss of MEPs was encountered in $50 \%$ of cases (50/ 99). In patients with loss of MEPs, $98 \%$ (49/50) had return of signals at the conclusion of the repair. The only patient whose MEPs did not return at the conclusion awoke paraplegic. In this case, the SSEPs remained unchanged. Of those cases where MEPs were lost, $44 \%$ (22/50) recovered with ICA reattachment, $32 \%(16 / 50)$ returned with pulsatile distal flow, $14 \%(7 / 50)$ returned with DAP, and $8 \%(4 / 50)$ returned spontaneously without any operative maneuvers.

In the 3 patients who experienced delayed neurologic deficit, both MEPs and SSEPs were lost in 2 cases but returned; in 1 case, no change was observed. In the 1 case of immediate paraplegia, the MEPs were lost and never returned. 


\section{Cerebrospinal Fluid Drainage Complications}

CSF complications occurred in $4 \%$ of cases (4/103). CSF catheter-associated intracranial hemorrhage occurred in 1 case $(1 \%)$ and was not associated with mortality. Three patients $(3 \%)$ had spinal headaches that resolved with medical management. One case of CSF drain malfunction was observed in a patient with delayed paraplegia; when the catheter was unkinked, this patient's neurologic function returned.

\section{DISCUSSION}

Our previous studies have demonstrated the efficacy of neuromonitoring using SSEPs and MEPs. ${ }^{3,4}$ In these studies, we found both SSEPs and MEPs to be specific $(97 \%)$ but not sensitive $(33 \%$ for SSEPs and $67 \%$ for MEPs, respectively). We acknowledged that a limitation to this work was related to the absence of an intraoperative control group, that is, alteration in the intraoperative conduct of the operation occurred on the basis of changes with either or both SSEPs and MEPs. A true control group would have included a subset of patients in whom no changes in intraoperative conduct occurred in response to neuromonitor changes. Acknowledging this, however, led us to more closely characterize the intraoperative maneuvers that were occurring and the results of neuromonitoring observed.

Several observations regarding neuromonitoring using SSEPs and MEPs are worth noting. Changes (loss) in MEPs predominantly occurred before any changes in SSEPs. In addition, return in SSEPs usually occurred before return in MEPs. MEPs were lost almost twice as frequently as SSEPs (50\% vs 26\%) during repair. Finally, intraoperative recovery of neurologic signals did occur with alterations in the conduct of the procedure, whether it was related to anesthesia or our intraoperative maneuvers. This emphasized the fact that alterations in procedural conduct could affect neuromonitoring signals.

Limitations regarding these observations require further discussion. Without a true control group for comparison, a definite correlation between neuromonitoring signal loss and spinal cord injury remains difficult to establish. However, the fact that the negative predictive valve of both SSEPs and MEPs was high $(97 \%-99 \%)^{3}$ indicated that some degree of correlation of SSEPs and MEPs with spinal cord injury exists. This suggests that SSEPs and MEPs are valuable during TAAA repairs. To determine the true sensitivity of SSEPs and MEPs during TAAA repair, however, avoidance of any corrective actions would have been required. Such a protocol would be difficult to justify ethically.

Another clarification pertains to the return of neuromonitoring signals, which was often attributed to an intraoperative maneuver closely associated with their recovery. In reality, many anesthetic measures (Table 2) were being carried out simultaneously with operative maneuvers, and the intraoperative maneuvers were not independent of other events that were also occurring at the same time. Thus, the return of signals was likely multifactorial. What cannot be dismissed, however, is that in approximately $50 \%$ of the cases in which signals returned, the recovery occurred immediately after ICA reattachment. This suggests that segmental artery reimplantation may be beneficial.

There is growing evidence that perfusion to the spinal cord is not dependent on a single vessel to the anterior spinal artery but rather on a complex collateral network. ${ }^{12}$ We previously demonstrated that the important segmental vessels include ICAs T8 to T12. ${ }^{13}$ In contrast, Griepp and Griepp ${ }^{14}$ reported that ICA reattachment may not be required, because the loss of segmental vessels may be compensated by perfusion to the collateral network by other means, such as increasing blood pressure.

This study provides evidence that ICA reimplantation may be beneficial. Although the study is limited by the fact that changes in MEP and SSEP signals do not absolutely correlate with spinal cord injury, it nevertheless stands to reason that even if the artery of Adamkiewicz is not as crucial to spinal cord perfusion as previously perceived, any added increase in perfusion to the collateral network (eg, ICA reattachment) should be advantageous. Thus, it remains reasonable to reattach segmental vessels when feasible and indicated, and not to rely on only anesthetic measures to improve overall perfusion to the collateral network.

We did not experience any differences in the occurrence of CSFD complications during the course of the study. Complications included 1 patient with an intracerebral hemorrhage and 3 patients with spinal headaches. One drain malfunction occurred as the result of kinking and led to delayed paraplegia, but this resolved after the CSF drain was straightened and the COPS protocol was initiated.

The incidence of paraplegia was similar to our previously reported incidence: $1 \%$ immediate and $3 \%$ delayed. ${ }^{5}$ It remains uncertain whether neuromonitoring-guided TAAA repair has affected paraplegia because this cohort remains small. It is notable, however, that the distribution of type of paraplegia has changed since the institution of adjuncts: Currently, most neurologic injuries are delayed as opposed to immediate. We previously demonstrated that an independent predictor for delayed spinal cord injury was the use of combined adjuncts. ${ }^{15,16}$ The likely explanation is that the use of adjuncts allowed patients to tolerate the initial insult of TAAA repair, but if other events occurred postoperatively, such as CSF drain malfunction or hypotension, then delayed paraplegia occurred. ${ }^{15}$ Thus, although an overall reduction in immediate paraplegia was observed, a relative increase in delayed paraplegia was experienced.

\section{CONCLUSIONS}

Neuromonitoring using SSEPs and MEPs seems useful during TAAA repair. Alterations in intraoperative conduct resulted in return of neuromonitoring signals. This suggests 
a benefit in ICA reimplantation, allowing increased perfusion to the collateral network of the spinal cord. Further studies using neuromonitoring-guided repair of TAAA are warranted.

The authors thank G. Ken Goodrick for editing and Chris Akers for the figures.

\section{References}

1. Svensson LG, Crawford ES, Hess KR, Coselli JS, Safi HJ. Experience with 1509 patients undergoing thoracoabdominal aortic operations. J Vasc Surg. 1993;17: 357-68.

2. Elefteriades JA. Editorial comment: motor evoked potentials in thoracoabdominal aortic surgery. Cardiol Clin. 2010;28:369-70.

3. Keyhani K, Miller CC III, Estrera AL, Wegryn T, Sheinbaum R, Safi HJ. Analysis of motor and somatosensory evoked potentials during thoracic and thoracoabdominal aortic aneurysm repair. J Vasc Surg. 2009;49: 36-41.

4. Achouh PE, Estrera AL, Miller CC III, Azizzadeh A, Irani A, Wegryn TL, et al. Role of somatosensory evoked potentials in predicting outcome during thoracoabdominal aortic repair. Ann Thorac Surg. 2007;84:782-8.

5. Safi HJ, Estrera AL, Miller CC, Huynh TT, Porat EE, Azizzadeh A, et al. Evolution of risk for neurologic deficit after descending and thoracoabdominal aortic repair. Ann Thorac Surg. 2005;80:2173-9.

6. Estrera AL, Sheinbaum R, Miller CC, Azizzadeh A, Walkes JC, Lee TY, et al. Cerebrospinal fluid drainage during thoracic aortic repair: safety and current management. Ann Thorac Surg. 2009;88:9-15.
7. Miller CC III, Villa MA, Sutton J, Lau D, Keyhani K, Estrera AL, et al. Serum myoglobin and renal morbidity and mortality following thoracic and thoracoabdominal aortic repair: does rhabdomyolysis play a role? Eur J Vasc Endovasc Surg. 2009;37:388-94.

8. Crawford ES, Mizrahi EM, Hess KR, Coselli JS, Safi HJ, Patel VM. The impact of distal aortic perfusion and somatosensory evoked potential monitoring on prevention of paraplegia after aortic aneurysm operation. J Thorac Cardiovasc Surg. 1988;95:357-67.

9. Griepp RB. Cerebral protection during aortic arch surgery. J Thorac Cardiovasc Surg. 2001;121:425-7.

10. Koeppel TA, Mess WH, Jacobs MJ. Motor evoked potentials in thoracoabdominal aortic surgery: PRO. Cardiol Clin. 2010;28:351-60.

11. Coselli JS, Tsai PI. Motor evoked potentials in thoracoabdominal aortic surgery: CON. Cardiol Clin. 2010;28:361-8.

12. Etz CD, Halstead JC, Spielvogel D, Shahani R, Lazala R, Homann TM, et al Thoracic and thoracoabdominal aneurysm repair: is reimplantation of spinal cord arteries a waste of time? Ann Thorac Surg. 2006;82:1670-7.

13. Safi HJ, Miller CC III, Carr C, Iliopoulos DC, Dorsay DA, Baldwin JC. Importance of intercostal artery reattachment during thoracoabdominal aortic aneurysm repair. J Vasc Surg. 1998;27:58-66.

14. Griepp RB, Griepp EB. Spinal cord perfusion and protection during descending thoracic and thoracoabdominal aortic surgery: the collateral network concept. Ann Thorac Surg. 2007;83:S865-9.

15. Estrera AL, Miller CC III, Huynh TT, Azizzadeh A, Porat EE, Vinnerkvist A et al. Preoperative and operative predictors of delayed neurologic deficit following repair of thoracoabdominal aortic aneurysm. J Thorac Cardiovasc Surg. 2003; 126:1288-94.

16. Azizzadeh A, Huynh TT, Miller CC III, Estrera AL, Porat EE, Sheinbaum R, et al. Postoperative risk factors for delayed neurologic deficit after thoracic and thoracoabdominal aortic aneurysm repair: a case-control study. J Vasc Surg. 2003;37:750-4. 